\title{
The MOSDEF Survey: First Measurement of Nebular Oxygen Abundance at $z>4^{*}$
}

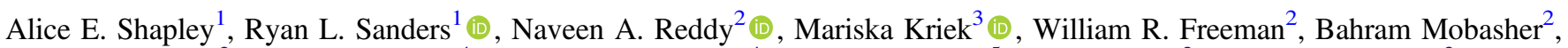 \\ Brian Siana ${ }^{2}$ (D), Alison L. Coil ${ }^{4}$ (D), Gene C. K. Leung ${ }^{4}$ (D) Laura deGroot ${ }^{5}$, Irene Shivaei ${ }^{2}$ (D), Sedona H. Price ${ }^{3}$ (D), \\ Mojegan Azadi ${ }^{4}$ (D), and James Aird ${ }^{6}$ (iD \\ ${ }^{1}$ Department of Physics and Astronomy, University of California, Los Angeles, 430 Portola Plaza, Los Angeles, CA 90095, USA; aes@astro.ucla.edu \\ ${ }^{2}$ Department of Physics and Astronomy, University of California, Riverside, 900 University Avenue, Riverside, CA 92521, USA \\ ${ }_{3}$ Astronomy Department, University of California at Berkeley, Berkeley, CA 94720, USA \\ ${ }^{4}$ Center for Astrophysics and Space Sciences, Department of Physics, University of California, San Diego, 9500 Gilman Drive, La Jolla, CA 92093, USA \\ ${ }^{5}$ Department of Physics, The College of Wooster, 1189 Beall Avenue, Wooster, OH 44691, USA \\ ${ }^{6}$ Institute of Astronomy, University of Cambridge, Madingley Road, Cambridge CB3 0HA, UK \\ Received 2017 July 15; revised 2017 August 23; accepted 2017 August 23; published 2017 September 8
}

\begin{abstract}
We present the first spectroscopic measurement of multiple rest-frame optical emission lines at $z>4$. During the MOSFIRE Deep Evolution Field survey, we observed the galaxy GOODSN-17940 with the Keck I/MOSFIRE spectrograph. The $K$-band spectrum of GOODSN-17940 includes significant detections of the [O II] $] \lambda 3726,3729$, [Ne III] $\lambda 3869$, and $\mathrm{H} \gamma$ emission lines and a tentative detection of $\mathrm{H} \delta$, indicating $z_{\text {spec }}=4.4121$. GOODSN-17940 is an actively star-forming $z>4$ galaxy based on its $K$-band spectrum and broadband spectral energy distribution. A significant excess relative to the surrounding continuum is present in the Spitzer/IRAC channel 1 photometry of GOODSN-17940, due primarily to strong $\mathrm{H} \alpha$ emission with a rest-frame equivalent width of $\mathrm{EW}(\mathrm{H} \alpha)=1200 \AA$. Based on the assumption of $0.5 Z_{\odot}$ models and the Calzetti attenuation curve, GOODSN-17940 is characterized by $M_{*}=5.0_{-0.2}^{+4.3} \times 10^{9} M_{\odot}$. The Balmer decrement inferred from $\mathrm{H} \alpha / \mathrm{H} \gamma$ is used to dust correct the $\mathrm{H} \alpha$ emission, yielding $\mathrm{SFR}(\mathrm{H} \alpha)=320_{-140}^{+190} M_{\odot} \mathrm{yr}^{-1}$. These $M_{*}$ and star formation rate (SFR) values place GOODSN-17940 an order of magnitude in SFR above the $z \sim 4$ star-forming "main sequence." Finally, we use the observed ratio of $[\mathrm{Ne} \mathrm{III}] /[\mathrm{O} \mathrm{II}]$ to estimate the nebular oxygen abundance in GOODSN-17940, finding $\mathrm{O} / \mathrm{H} \sim 0.2(\mathrm{O} / \mathrm{H})_{\odot}$. Combining our new $[\mathrm{Ne} \mathrm{III}] /[\mathrm{O} \mathrm{II}]$ measurement with those from stacked spectra at $z \sim 0,2$, and 3 , we show that GOODSN-17940 represents an extension to $z>4$ of the evolution toward higher [Ne III]/[O II] (i.e., lower O/H) at fixed stellar mass. It will be possible to perform the measurements presented here out to $z \sim 10$ using the James Webb Space Telescope.
\end{abstract}

Key words: galaxies: evolution - galaxies: high-redshift - galaxies: ISM

\section{Introduction}

The study of high-redshift galaxies has been transformed by major recent advances in infrared instrumentation. New multiobject near-IR spectrographs on 8-10 m class ground-based telescopes have enabled ground-breaking discoveries about galaxies at $z \sim 1.5-3.5$, the epoch of peak star formation and black hole accretion activity (Madau \& Dickinson 2014). In particular, statistical samples of rest-frame optical spectra collected with the MOSFIRE spectrograph on Keck I (McLean et al. 2012) and KMOS spectrograph on the VLT (Sharples et al. 2013) have yielded detailed probes of the gas, dust, heavy elements, stars, supermassive black holes, and dynamics in galaxies at high redshift-all of which are essential to understanding the basic questions of galaxy formation.

However, there are virtually no direct rest-frame optical spectroscopic measurements beyond $z=4$. At such redshifts, the strongest rest-frame optical emission lines (e.g., $\mathrm{H} \alpha$ and $[\mathrm{O}$ III $] \lambda 5007)$ are shifted into the thermal infrared and inaccessible from the ground. The strengths of these lines have only been inferred indirectly at $z \sim 4-7$ based on Spitzer/

\footnotetext{
* Based on data obtained at the W.M. Keck Observatory, which is operated as a scientific partnership among the California Institute of Technology, the University of California, and the National Aeronautics and Space Administration, and was made possible by the generous financial support of the W.M. Keck Foundation.
}

IRAC photometric measurements (e.g., Stark et al. 2013; Smit et al. 2014). In addition, spectroscopic detections of the [O II] $\lambda \lambda 3726,3729$ doublet have been presented for only three gravitationally lensed galaxies, all at $z=4.9$ (Swinbank et al. 2007, 2009; Troncoso et al. 2014).

The launch of the James Webb Space Telescope (JWST) will enable the extension of rest-frame optical spectroscopic probes of the contents of galaxies from the peak epoch of star formation out to $z=4-10$. Meanwhile, in the course of the MOSFIRE Deep Evolution Field (MOSDEF) survey (Kriek et al. 2015), we have serendipitously collected the rest-frame optical spectrum of an unlensed galaxy in the GOODS-N field at $z=4.4121$. The analysis of this galaxy, GOODSN-17940, provides the first rest-frame optical nebular abundance estimate at $z>4$ and a preview of the advances soon to come with JWST. In Section 2, we describe our observations and data analysis. In Section 3, we present the MOSFIRE $K$-band spectrum of GOODSN-17940 and determine several key physical properties including the nebular oxygen abundance in Section 4. Finally, in Section 5, we consider an active galactic nucleus (AGN) contribution to GOODSN-17940, place its oxygen abundance measurement into the context of the global chemical evolution of star-forming galaxies, and consider future rest-frame optical spectroscopic observations of GOODSN17940 and other $z>4$ star-forming galaxies. Throughout, we adopt cosmological parameters of $H_{0}=70 \mathrm{~km} \mathrm{~s}^{-1} \mathrm{Mpc}^{-1}$, $\Omega_{M}=0.3$, and $\Omega_{\Lambda}=0.7$. 

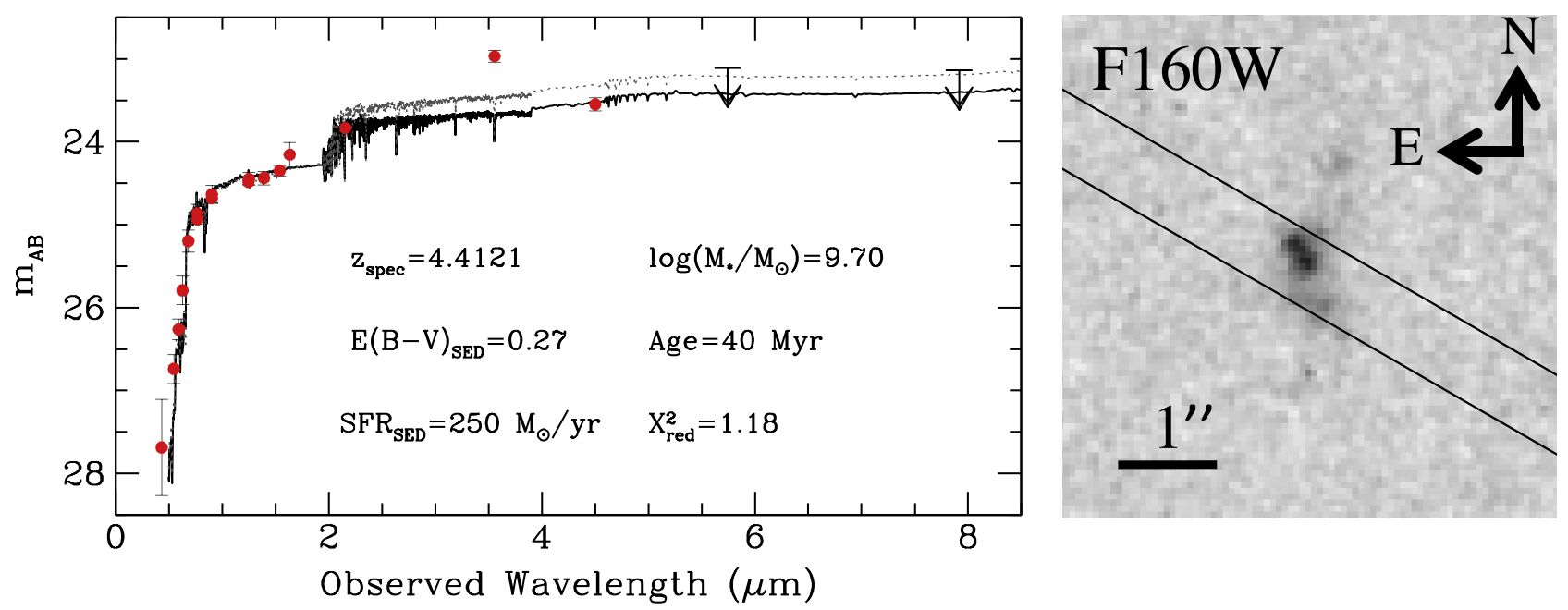

Figure 1. Stellar populations and image of GOODSN-17940. Left: the observed and best-fit model SEDs for GOODSN-17940. Photometric detections are indicated with red symbols, including the IRAC channel 1 data point, which suggests significant contamination by nebular emission. We plot $2 \sigma$ limits for the IRAC channel 3 and 4 non-detections. The best-fit stellar population model of GOODSN-17940, excluding IRAC channel 1, is shown as the solid black curve. We also list the parameters of this model from Conroy et al. (2009), which assumes $Z=0.5 Z_{\odot}$ and a Calzetti attenuation law. The best-fit model to the SED including IRAC channel 1 is shown as a dotted gray curve. This fit is biased high by a factor of 2.7 in stellar mass relative to the clean fit. Right: WFC3/F160W postage stamp image of GOODSN-17940, with the 0!! 7 MOSFIRE slit overlaid. At $z=4.4121$, the F160W filter probes $\lambda_{\text {rest }}=2840 \AA$. GOODSN-17940 consists of two central clumps separated by $\sim 1 \mathrm{kpc}$ (proper), surrounded by an irregular, lower surface brightness region that extends toward the south. The emission located roughly $1^{\prime \prime}$ to the northwest is identified as a separate object in the 3D-HST photometric catalog (ID $\left.=17884, z_{\text {phot }}=3.93\right)$.

\section{Observations}

GOODSN-17940 (R.A. $=12: 36: 35.49$ and decl. $=62: 13: 50.0$ (J2000), $\left.m_{\mathrm{F} 160 \mathrm{~W}, \mathrm{AB}}=24.35\right)$ was observed as part of the MOSDEF survey (Kriek et al. 2015). MOSDEF is a large survey of the rest-frame optical spectra of $\sim 1500$ galaxies at $1.4 \leqslant$ $z \leqslant 3.8$. We collected Keck I/MOSFIRE data for MOSDEF over 48.5 nights spanning from 2012 December through 2016 May. MOSFIRE $H$ - and $K$-band spectroscopic data were obtained for a multi-object slitmask containing GOODSN-17940 on 2016 March 18. The slit width was 0.7 , yielding a spectral resolution of $\sim 3650$ in $H$ and $\sim 3600$ in $K$. The total integration time in both $H$ and $K$ was 120 minutes. The conditions were clear with a median seeing of 0 "! 6 .

We reduced the raw data to produce two-dimensional science and error spectra using a custom IDL pipeline. We then optimally extracted one-dimensional science and error spectra from the two-dimensional spectra, applying slit-loss corrections as described in Kriek et al. (2015). Our interpretation of GOODSN-17940 is greatly enhanced by the extensive multi-wavelength coverage in the GOODS-N field, including broadband photometry in 22 filters spanning from the near-UV through mid-infrared (Skelton et al. 2014; Momcheva et al. 2016).

MOSDEF targets galaxies in three discrete redshift intervals, $1.37 \leqslant z \leqslant 1.70, \quad 2.09 \leqslant z \leqslant 2.61, \quad$ and $\quad 2.95 \leqslant z \leqslant 3.80$ (Kriek et al. 2015). GOODSN-17940 was targeted based on a prior spectroscopic redshift of $z_{\mathrm{spec}}=3.665$, which placed it in the most distant MOSDEF target redshift interval. This redshift was derived from Keck/DEIMOS spectroscopy and the putative detection of Ly $\alpha$ at $5673 \AA$, which was unconfirmed when recently re-examined (S. Hemmati 2017, private communication). The photometric redshift of GOODSN-17940 in the publicly available 3D-HST catalog is $z_{\text {phot }}=4.447$ (Momcheva et al. 2016), consistent with the one measured from our MOSFIRE $K$-band spectrum. We also note that another Keck/DEIMOS spectrum nominally targeting the fainter, diffuse morphological component $0 . " 6$ to the south (see Figure 1, right) yielded a secure detection of Ly $\alpha$ at $z=4.416$ (M. Dickinson 2017, private communication).

\section{The Spectrum of GOODSN-17940}

As shown in Figure 2, the MOSFIRE $K$-band spectrum of GOODSN-17940 includes significant detections of the [O II] $\lambda \lambda 3726,3729$, [Ne III] $\lambda 3869$, and $\mathrm{H} \gamma$ emission lines, along with a tentative $2.3 \sigma$ detection of $\mathrm{H} \delta$. Based on the observed centroid of the highest signal-to-noise ratio $(\mathrm{S} / \mathrm{N})$ feature, [Ne III] $\lambda 3869$, we estimate a spectroscopic redshift of $z_{\text {spec }}=4.4121$. We fit Gaussian profiles to the emission lines, using a single-component model for [Ne III] $\lambda 3869$ and the Balmer lines and a two-component model for [O II] $\lambda \lambda 3726,3729$. The ratio of wavelengths was fixed for the two [O II] doublet members, and their profile FWHMs were forced to be identical. Uncertainties on reported quantities were estimated using Monte Carlo simulations, in which 500 perturbed fake spectra were generated from the observed spectrum according to the error spectrum, and the measurements repeated on each fake spectrum. The $1 \sigma$ confidence intervals for each measurement were determined from the distributions of simulated best-fit parameters.

Observed line fluxes and uncertainties are listed in Table 1. We note that both the [Ne III] and [O II] emission lines are well-resolved spectrally, with the [Ne III] line suggesting a line width (corrected for instrumental broadening) of $\sigma_{v}=160_{-50}^{+10} \mathrm{~km} \mathrm{~s}^{-1}$. The Balmer features are not well-resolved in the spectral dimension, which may be a result of their lower $\mathrm{S} / \mathrm{N}$. Finally, while the density-sensitive [O II] doublet is blended and only of $4.7 \sigma$ significance, our two-component fitting simulations suggest that the bluer doublet member is the stronger one, corresponding to the high-density limit (Sanders et al. 2016b). 


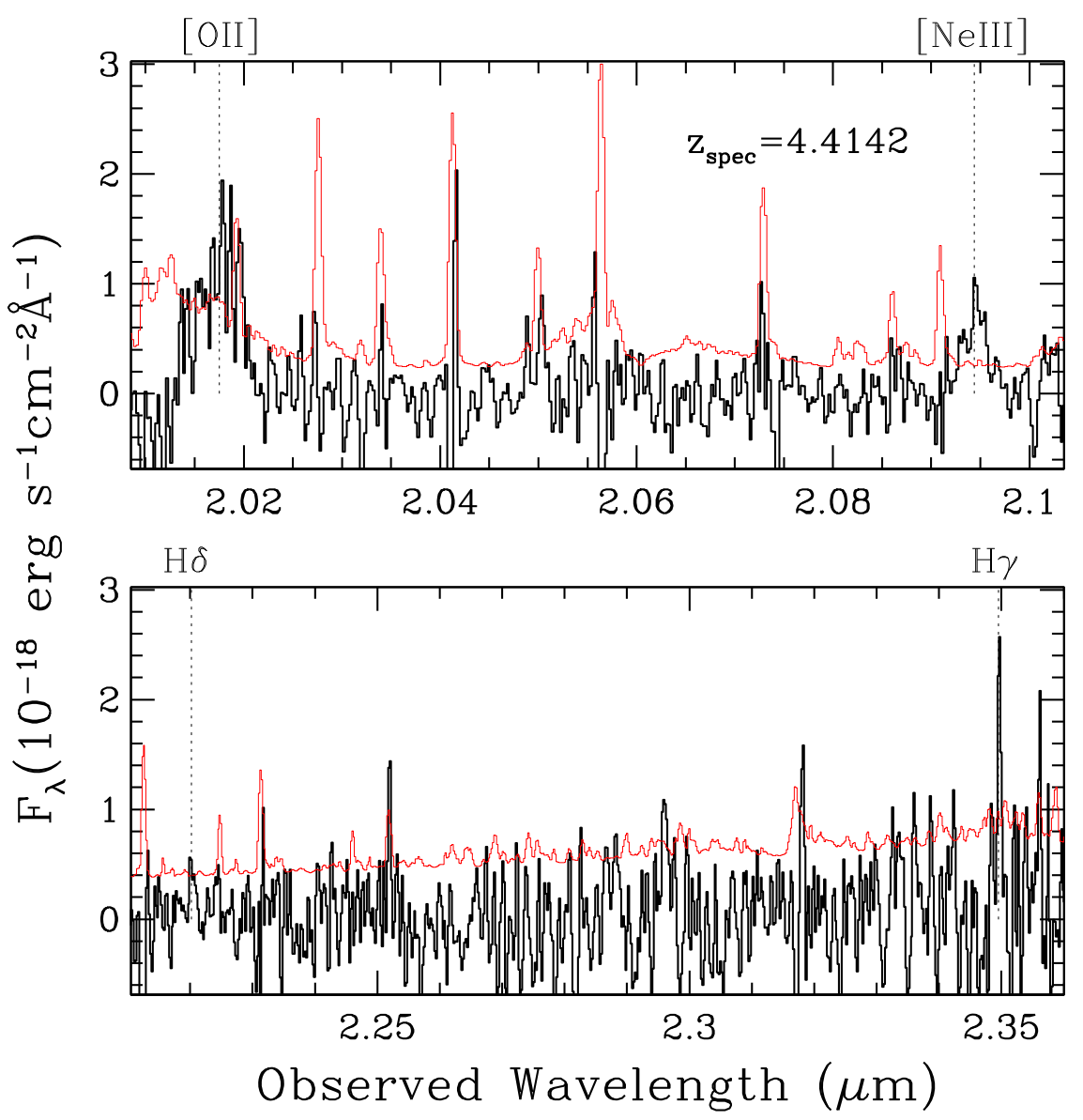

Figure 2. Observed-frame MOSFIRE $K$-band spectrum of GOODSN-17940. Flux density units are $10^{-18} \mathrm{erg} \mathrm{s}^{-1} \mathrm{~cm}^{-2} \AA^{-1}$. The error spectrum is overplotted in red, and the locations of $[\mathrm{O}$ II] $\lambda \lambda 3726,3729,[\mathrm{Ne}$ III] $] 33869, \mathrm{H} \delta$, and $\mathrm{H} \gamma$ are labeled and marked with dotted vertical lines. The redshift we report from the highest $\mathrm{S} / \mathrm{N}$ feature, [Ne III], is consistent with those estimated from the other labeled features.

Table 1

Emission-line Properties of GOODSN-17940a

\begin{tabular}{lc}
\hline \hline Line & Flux \\
\hline$[\mathrm{O}$ II $] \lambda \lambda 3726,3729$ & $6.9 \pm 1.5$ \\
{$[\mathrm{Ne}$ III] $] 33869$} & $2.2 \pm 0.5$ \\
$\mathrm{H} \delta$ & $0.7 \pm 0.3$ \\
$\mathrm{H} \gamma$ & $2.1 \pm 0.5$ \\
\hline
\end{tabular}

Note.

${ }^{\text {a }}$ Observed emission-line flux in units of $10^{-17} \mathrm{erg} \mathrm{s}^{-1} \mathrm{~cm}^{-2}$.

\section{The Physical Properties of GOODSN-17940}

The combination of the MOSFIRE $K$-band spectrum, broadband spectral energy distribution (SED), and Hubble Space Telescope imaging of GOODSN-17940 provides a window into a remarkable $z \sim 4$ galaxy, including its stellar population, dust content, current star formation rate (SFR), and nebular oxygen abundance.

\subsection{Stellar Population}

Figure 1 (left) shows the observed SED of GOODSN-17940. There is evidence for strong $\mathrm{H} \alpha+[\mathrm{N}$ II $] \lambda \lambda 6548,6584+[\mathrm{S} \mathrm{II}]$ $\lambda \lambda 6717,6731$ emission-line contamination in the Spitzer/IRAC channel 1 data point at $3.6 \mu \mathrm{m}$, as this measurement is significantly elevated relative to the surrounding data points at the $K$ band and IRAC channel 2 . In order to quantify both stellar population parameters and nebular emission flux within IRAC channel 1, we modeled the broadband SED of GOODSN-17940 using the fitting code, FAST (Kriek et al. 2009). In this analysis, we excluded the contaminated IRAC channel 1 data point and adopted the population synthesis models of Conroy et al. (2009), the Calzetti et al. (2000) attenuation curve, and a Chabrier (2003) initial mass function (IMF). We further assumed a delayed exponential star formation history of the form SFR $\propto t \exp (-t / \tau)$, with $t$ as the time since the onset of star formation and $\tau$ as the characteristic timescale for the decay of star formation. Based on the inferred oxygen abundance of GOODSN-17940 described in Section 4.5, we used model spectra with 0.5 solar abundance $\left(Z=0.5 Z_{\odot}\right)$, the lowest-metallicity default option in FAST for the Conroy et al. (2009) models.

The SED modeling for GOODSN-17940 results in best-fit parameters of $M_{*}=5.0_{-0.2}^{+4.3} \times 10^{9} M_{\odot}, E(B-V)_{\mathrm{SED}}=0.27_{-0.05}^{+0.0}$, $\mathrm{SFR}_{\mathrm{SED}}=250_{-140}^{+20} M_{\odot} \mathrm{yr}^{-1}$, and a young age $(\leqslant 200 \mathrm{Myr})$. Figure 1 shows the best-fit models both excluding (black solid) and including (gray dashed) IRAC channel 1. In the latter case, the best-fit stellar mass is biased toward a higher value of $1.3 \times 10^{10} M_{\odot}$. We note that the best-fit stellar population parameters for GOODSN-17940 depend systematically on the input model assumptions. For example, if we instead follow 
Reddy et al. (2017) and fit GOODSN-17940 with a $Z=0.14 Z_{\odot}$ BPASS model (Stanway et al. 2016) that includes massive stellar binaries, nebular continuum emission, and a constant star formation history, and we assume a Small Magellanic Cloud (SMC) attenuation curve (Gordon et al. 2003), we find $M_{*}=1.3 \times 10^{10} M_{\odot}, \quad E(B-V)_{\mathrm{SED}}=0.10, \quad \mathrm{SFR}_{\mathrm{SED}}=$ $30 M_{\odot} \mathrm{yr}^{-1}$, and Age $=500 \mathrm{Myr}$. Accordingly, these other assumptions yield a higher stellar mass for GOODSN-17940, and lower SFR and specific SFR $\left(\mathrm{SFR} / M_{*}\right)$. More work is needed to determine the optimal SED modeling assumptions for $z>4$ galaxies such as GOODSN-17940. In what follows, we interpret GOODSN-17940 using the $Z=0.5 Z_{\odot}$ Conroy et al. model, but we highlight systematic uncertainties related to SED fitting when relevant.

\section{2. $H \alpha$ Emission}

The observed excess at IRAC channel 1 relative to the bestfit SED model excluding this data point can be used to estimate the nebular emission flux, $F_{\mathrm{em}}$, causing the excess. Specifically,

$$
F_{\mathrm{em}}=\Delta \lambda\left(F_{\lambda, \mathrm{ch} 1, \mathrm{obs}}-F_{\lambda, \mathrm{ch} 1, \text { model }}\right),
$$

where $\Delta \lambda=7500 \AA$ is the width of the IRAC channel 1 filter, $F_{\lambda, \text { ch } 1, \text { obs }}=5.53_{-0.03}^{+0.03} \times 10^{-20} \mathrm{erg} \mathrm{s}^{-1} \mathrm{~cm}^{-2} \AA^{-1}$ is the observed IRAC channel 1 flux density, and $F_{\lambda \text {,ch1,model }}=2.93_{-0.01}^{+0.03} \times$ $10^{-20} \mathrm{erg} \mathrm{s}^{-1} \mathrm{~cm}^{-2} \AA^{-1}$ is the IRAC channel 1 flux density in the best-fit SED model excluding the IRAC channel 1 data point. Based on the oxygen abundance in the ionized interstellar medium (ISM) of GOODSN-17940 discussed below, we further assume that the nebular emission is dominated by $\mathrm{H} \alpha$, and make the approximation $F_{\mathrm{em}} \approx F(\mathrm{H} \alpha)$. We find that $F(\mathrm{H} \alpha)=$ $2.0_{-0.3}^{+0.2} \times 10^{-16} \mathrm{erg} \mathrm{s}^{-1} \mathrm{~cm}^{-2}$, which corresponds to a rest-frame equivalent width of $\operatorname{EW}(\mathrm{H} \alpha)=1200_{-200}^{+100} \AA$. This $\operatorname{EW}(\mathrm{H} \alpha)$ is significantly larger than the average of $\sim 400 \AA$ found by Stark et al. (2013) for a sample of 92 spectroscopically confirmed starforming galaxies at $3.8 \leqslant z \leqslant 5.0$ and demonstrates the extreme nature of GOODSN-17940. Based on our analysis, the contribution from nebular line emission in GOODSN-17940 is slightly less than $50 \%$ of the total flux in the IRAC channel 1 band. Both the inferred $F(\mathrm{H} \alpha)$ and $\mathrm{EW}(\mathrm{H} \alpha)$ are insensitive to the choice of SED fitting assumptions.

\subsection{Dust Extinction}

We combine $F(\mathrm{H} \alpha)$ inferred from the IRAC channel 1 excess and $F(\mathrm{H} \gamma)$ measured directly from the MOSFIRE $K$-band spectrum to estimate the Balmer decrement. The theoretical $F(\mathrm{H} \alpha) / F(\mathrm{H} \gamma)$ ratio is 6.11 , based on the assumption of a temperature of $T=10,000 \mathrm{~K}$, an electron density of $n_{\mathrm{e}}=100 \mathrm{~cm}^{-3}$, and Case B recombination. The observed ratio of $F(\mathrm{H} \alpha) / F(\mathrm{H} \gamma)_{\mathrm{obs}}=9.3_{-2.4}^{+3.1}$ then implies $E(B-V)_{\text {gas }}=0.28 \pm 0.19$, with the assumption of the Cardelli et al. (1989) dust attenuation curve. In this calculation, we have not corrected $\mathrm{H} \alpha$ and $\mathrm{H} \gamma$ fluxes for underlying stellar Balmer absorption. Based on the best-fit stellar population model for GOODSN-17940, we find that the $\mathrm{H} \gamma$ absorption correction is $\sim 1 \%$ of the measured $\mathrm{H} \gamma$ emission flux, while the fractional contribution of the Balmer absorption correction to $\mathrm{H} \alpha$ is an order of magnitude smaller. Therefore, the application of such corrections makes no significant difference to our results.

\subsection{Current Star Formation}

We apply the inferred $E(B-V)_{\text {gas }}$ to calculate the dustcorrected $\mathrm{H} \alpha$ flux and SFR. The dust-corrected $\mathrm{H} \alpha$ flux is $F(\mathrm{H} \alpha)=3.7_{-1.6}^{+2.0} \times 10^{-16} \mathrm{erg} \mathrm{s}^{-1} \mathrm{~cm}^{-2}$, which corresponds to $\mathrm{SFR}(\mathrm{H} \alpha)=320_{-140}^{+190} M_{\odot} \mathrm{yr}^{-1}$ at $z=4.4121$. Here, we assume the Kennicutt (1998) calibration between $\mathrm{H} \alpha$ luminosity and SFR, applying a normalization factor of 1.8 to convert to a Chabrier (2003) IMF. The Kennicutt calibration likely overestimates $\operatorname{SFR}(\mathrm{H} \alpha)$ by up to a factor of $\sim 2$ for GOODSN17940 , given that this calibration is based on solar metallicity models, while the metallicity we infer in Section 4.5 for GOODSN-17940 is subsolar. As shown in Stanway et al. (2016), subsolar stellar population models produce more ionizing photons per unit solar mass of star formation. Regardless, $\operatorname{SFR}(\mathrm{H} \alpha)$ is consistent within $1 \sigma$ with $\operatorname{SFR}_{\mathrm{SED}}=$ $250_{-140}^{+20} M_{\odot} \mathrm{yr}^{-1}$ inferred from the best-fit stellar population model. Both methods indicate that GOODSN-17940 is highly star forming and significantly elevated in SFR relative to the "main sequence" correlation between SFR and $M_{*}$ at $z=4-5$ (e.g., Salmon et al. 2015). GOODSN-17940 instead falls within the $z=4-5$ "starburst" cloud recently identified by Caputi et al. (2017).

\subsection{Nebular Oxygen Abundance}

As highlighted by Nagao et al. (2006), the [Ne III] $\lambda 3869 /$ [O II] $\lambda \lambda 3726,3729$ ratio declines monotonically with increasing metallicity. The measurement of both [Ne III] $\lambda 3869$ and [O II] $\lambda \lambda 3726,3729$ can therefore be used to estimate the nebular oxygen abundance in GOODSN-17940. Both Maiolino et al. (2008) and Jones et al. (2015) have presented calibrations between $[\mathrm{Ne} \mathrm{III}] /[\mathrm{O} \mathrm{II}]$ and $12+\log (\mathrm{O} / \mathrm{H})$. Jones et al. (2015) restricts the calibration of $[\mathrm{Ne} \mathrm{III}] /[\mathrm{O} \mathrm{II}]$ to the low-metallicity regime in which direct oxygen abundances are available $(12+\log (\mathrm{O} / \mathrm{H}) \leqslant 8.4)$, while Maiolino et al. (2008) extends this calibration into the metal-rich regime using $12+\log (\mathrm{O} / \mathrm{H})$ estimates based on photoionization modeling. The measured $[\mathrm{Ne} \mathrm{III}] /[\mathrm{O} \mathrm{II}]$ ratio is $F([\mathrm{Ne} \mathrm{III}]) / F([\mathrm{O} \mathrm{II}])=0.33_{-0.08}^{+0.10}$, not including a dust correction due to the proximity of [Ne III] $\lambda 3869$ and [O II] $\lambda \lambda 3726,3729$ in wavelength. This ratio corresponds to $12+\log (\mathrm{O} / \mathrm{H})=7.89_{-0.14}^{+0.15}\left[8.05_{-0.06}^{+0.06}\right]$ according to the calibrations of Maiolino et al. [Jones et al.]. Both $[\mathrm{Ne}$ III] $/[\mathrm{O}$ II] calibrations suggest that GOODSN-17940 has O/ $\mathrm{H} \sim 0.2(\mathrm{O} / \mathrm{H})_{\odot}$ (Asplund et al. 2009).

There has been much recent discussion in the literature regarding the applicability (or lack thereof) of strong-line metallicity calibrations at $z \geqslant 2$, in particular those including the $[\mathrm{N}$ II $] \lambda 6584 / \mathrm{H} \alpha$ ratio (Masters et al. 2014; Steidel et al. 2014; Shapley et al. 2015; Sanders et al. 2016b). However, Jones et al. (2015) and Sanders et al. (2016a) present evidence that the relationship between $[\mathrm{Ne} \mathrm{III}] /[\mathrm{O}$ II $]$ and directly determined $12+\log (\mathrm{O} / \mathrm{H})$ does not evolve strongly out to $z \sim 3$. We therefore conclude that the $[\mathrm{Ne} \mathrm{III}] /[\mathrm{O} \mathrm{II}]$ indicator should provide a reliable estimate of the gas-phase metallicity in GOODSN-17940. Accordingly, our observations provide the first nebular oxygen abundance at $z>4$ based on a rest-frame optical spectrum.

In some respects, the significantly subsolar metallicity of GOODSN-17940 is unexpected. In the local universe, there is an observed correlation between dust extinction and metallicity (Heckman et al. 1998) in starburst galaxies, suggesting that the 

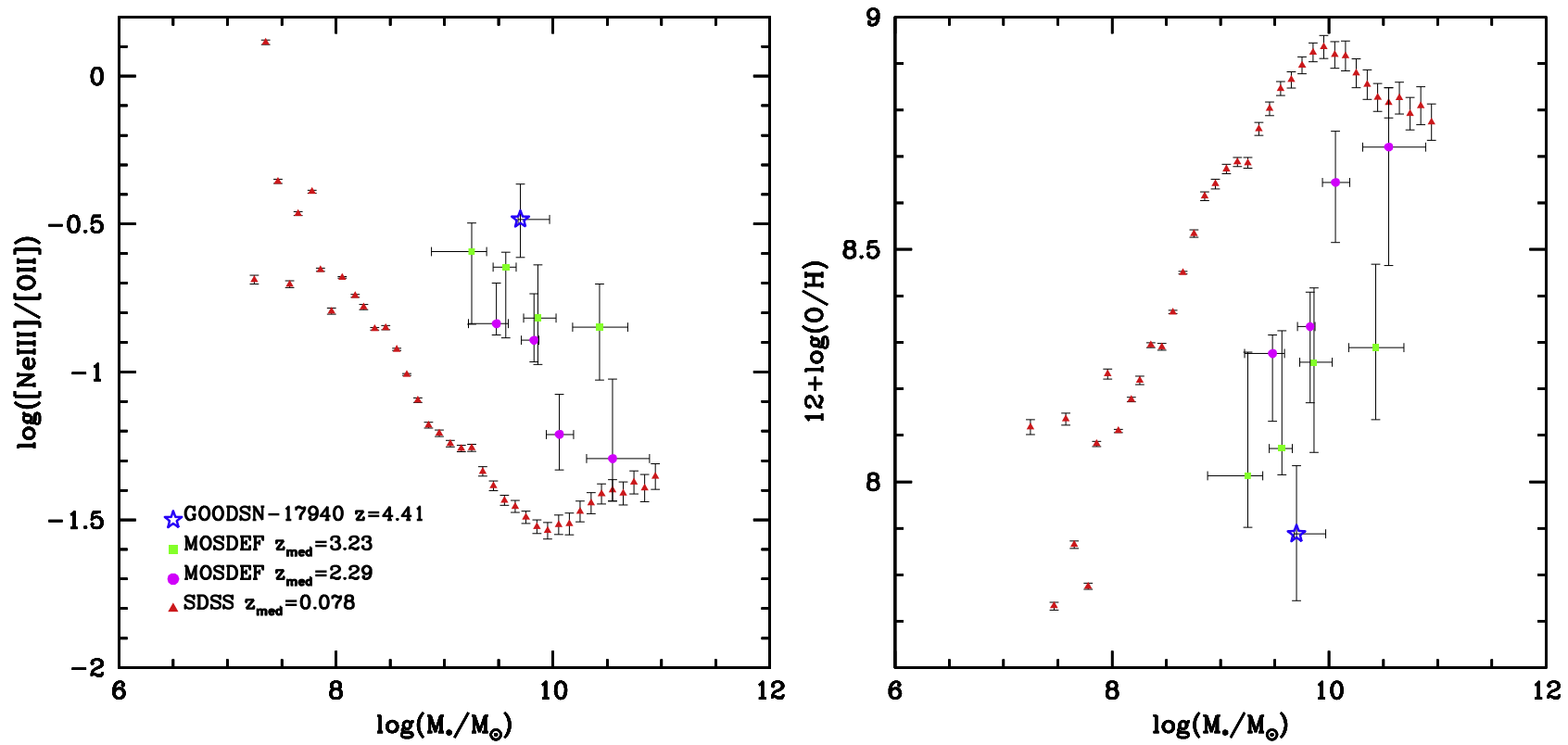

Figure 3. Evolution in the mass-metallicity relationship. Left: the observed ratio of [Ne III]/[O II] vs. stellar mass for composite spectra of SDSS galaxies of stellar mass at $z \sim 0$ (red triangles), composite spectra of MOSDEF samples at $z \sim 2$ and $z \sim 3$ (magenta circles and green squares, respectively), and GOODSN-17940 at $z=4.4121$ (large blue star). There is a clear progression toward higher [Ne III] $/[\mathrm{O}$ II] at fixed mass, which can be understood as an evolution toward lower oxygen abundance. Right: $12+\log (\mathrm{O} / \mathrm{H})$ vs. stellar mass, based on the [Ne III]/[O II] calibration of Maiolino et al. (2008). As a single, high-specific-SFR galaxy, GOODSN17940 may not be representative of the average $5 \times 10^{9} M_{\odot}$ galaxy at $z=4.4$, and its offset relative to the lower-redshift systems of similar mass is likely enhanced relative to more typical $z>4$ galaxies.

dust-to-gas ratio is correlated with the degree of chemical enrichment. Reddy et al. (2010) demonstrated that $z \sim 2$ starforming galaxies follow the same correlation between extinction and metallicity, with a similar fraction of metals locked in dust grains. Accordingly, if GOODSN-17940 followed the same pattern between extinction and metallicity, its significant dust reddening inferred from both the SED model shown in Figure 1 and the $\mathrm{H} \alpha / \mathrm{H} \gamma$ Balmer decrement would suggest solar, not $\sim 20 \%$ solar, metallicities. Investigating the connection between dust extinction and metallicity at $z>4$ with a larger sample will provide important insights into dust production mechanisms in the early universe and help us place GOODSN-17940 into context.

\section{Discussion}

\subsection{Is GOODSN-17940 an AGN?}

The line width measured from [Ne III], the highest $\mathrm{S} / \mathrm{N}$ feature in the GOODSN-17940 spectrum, is $\sigma_{v}=160 \mathrm{~km} \mathrm{~s}^{-1}$. The width of this feature is larger than those reported in studies of $z \sim 3$ ionized gas kinematics (e.g., Turner et al. 2017). Ionized gas associated with an AGN could cause a broad line profile. If the emission lines in the spectrum of GOODSN17940 originate from gas excited by the ionizing spectrum of an AGN rather than massive stars, our estimates of the nebular metallicity and SFR of GOODSN-17940 are invalid.

However, based on the current evidence, GOODSN-17940 is unlikely to host an AGN. The intense star formation in GOODSN-17940 suggests that it is a gas-rich system (Schmidt 1959). If the gas mass fraction in GOODSN-17940 is $\geqslant 60 \%$ (easily plausible; see, e.g., Tacconi et al. 2013), applying the virial theorem to the total baryonic mass and size of $\sim 1 \mathrm{kpc}$ yields a velocity dispersion consistent with the observed value. Furthermore, the extreme SFR surface density in GOODSN-17940 places it well above the threshold for driving significant gas outflows (Heckman 2002), which could broaden the nebular line profiles (e.g., Genzel et al. 2011). Finally, we find that GOODSN-17940 is undetected in the 2 Ms Chandra image of the GOODS-N field, with a rest-frame $2-10 \mathrm{keV}$ upper limit of $\log \left(L_{X} / \mathrm{erg} \mathrm{s}^{-1}\right)=42.68 \quad(95 \%$ confidence). In the future, measurements of the $\mathrm{H} \beta$, [O III] $\lambda 5007, \mathrm{H} \alpha$, and [N II] $\lambda 6584$ features with $J W S T$ will provide stronger constraints on possible AGN contribution.

\subsection{Evolution in the Mass-Metallicity Relation}

Over a wide range of redshifts, star-forming galaxies follow an evolving correlation between gas-phase oxygen abundance and stellar mass-the so-called mass-metallicity relation (MZR; e.g., Tremonti et al. 2004; Zahid et al. 2014; Sanders et al. 2015; Onodera et al. 2016). At a given stellar mass, the MZR evolves toward lower metallicity as redshift increases. With the measurement of $[\mathrm{Ne} \mathrm{III}] /[\mathrm{O} \mathrm{II}]$, we can place GOODSN-17940 in the context of the evolving MZR. For this analysis, we compare GOODSN-17940 with samples of star-forming galaxies at $z \sim 0,2$, and 3, both in the empirical sense of $[\mathrm{Ne} \mathrm{III}] /[\mathrm{O} \mathrm{II}]$ versus $M_{*}$ and the more physical dimensions of $12+\log (\mathrm{O} / \mathrm{H})$ versus $M_{*}$. For the local comparison sample, we use the $z \sim 0.08$ SDSS composite spectra of Andrews \& Martini (2013), which are binned by stellar mass. At higher redshift, we draw galaxies from the MOSDEF survey, again using composite spectra in bins of stellar mass. The two MOSDEF samples have median redshifts of $z=2.29$ and $z=3.23$. The [Ne III]/[O II] ratio and error are measured for each composite spectrum, as well as the median stellar mass in the bin. In Figure 3 (left), a clear progression is evident at fixed mass toward higher [Ne III]/[O II], as redshift increases from $z \sim 0$ to $z>4$. Adopting the Maiolino et al. (2008) calibration between $[\mathrm{Ne} \mathrm{III}] /[\mathrm{O} \mathrm{II}]$ and oxygen abundance (Figure 3, right), we see that the increase in [ $\mathrm{Ne} \mathrm{III]/}$ 
[O II] from $z=0-4$ at the mass of GOODSN-17940 corresponds to a decrease in $12+\log (\mathrm{O} / \mathrm{H})$ of $1.0 \mathrm{dex}$.

In the local universe, the MZR is observed to have a significant secondary dependence on SFR, such that, at fixed stellar mass, galaxies are offset toward lower metallicity at increasing SFRs. The relationship among metallicity, stellar mass, and SFR has been termed the "fundamental metallicity relation" (FMR; Mannucci et al. 2010) and has been interpreted using a scenario where infalling metal-poor gas both stimulates star formation and dilutes the metallicity. Results thus far are inconclusive regarding the existence of the FMR at high redshift (see, e.g., Belli et al. 2013; Sanders et al. 2015). However, if the FMR does persist out to $z \sim 4$, GOODSN17940 would be offset toward even lower metallicity than typical $z \sim 4$ star-forming galaxies of the same mass, given that its SFR is elevated by an order of magnitude relative to the $z \sim 4$ star-forming main sequence. Accordingly, the evolution in metallicity out to $z \sim 4$ may not be as extreme as GOODSN17940 suggests on its own. It will be possible to resolve this question based on statistical samples of $z \sim 4$ star-forming galaxies with stellar mass and metallicity measurements from $J W S T$.

\subsection{Summary and Outlook}

We have presented the first measurements of multiple restframe optical emission lines in the spectrum of a galaxy at $z>4$. Observed with Keck/MOSFIRE during the MOSDEF survey, this galaxy, GOODSN-17940, exhibits remarkable properties. Its active star formation places it significantly above the $z=4 \mathrm{SFR}-M_{*}$ main sequence, and, although showing evidence for considerable dust extinction, its nebular oxygen abundance is only $20 \%$ solar.

The thermal infrared background prevents us from obtaining longer-wavelength rest-frame optical spectra from the ground for GOODSN-17940. However, with the launch of JWST, it will become commonplace to analyze the rest-frame optical spectra of $z>4$ galaxies. GOODSN-17940 will make for a particularly compelling target, as it should exhibit detectable auroral [O III] $\lambda 4363$ emission. Such measurements enable a determination of the electron temperature, the so-called "direct" oxygen abundance, and therefore an empirical calibration between strong emission-line ratios and metallicity. We infer the detectability of $[\mathrm{O}$ III $] \lambda 4363$ by calculating the ratio of $[\mathrm{O}$ III $] \lambda 4363 / \mathrm{H} \gamma$ emission flux in the composite spectrum from Andrews \& Martini (2013) that shows the same $[\mathrm{Ne}$ III]/[O II] ratio as GOODSN-17940. Assuming the same [O III] $\lambda 4363 / \mathrm{H} \gamma$ ratio for GOODSN-17940, we predict $\mathrm{F}([\mathrm{O} \mathrm{III}] \lambda 4363)=4 \times 10^{-18} \mathrm{erg} \mathrm{s}^{-1} \mathrm{~cm}^{-2}$, which will be easily detectable with the $J W S T /$ NIRSpec instrument. Finally, we note that the spectroscopic measurements presented here will be possible out to $z \sim 10$ using JWST/NIRSpec, well into the reionization epoch.

We acknowledge support from NSF AAG grants AST1312780, 1312547, 1312764, and 1313171; grant AR-13907 from the Space Telescope Science Institute; and grant NNX16AF54G from the NASA ADAP program. J.A. acknowledges support from ERC Advanced Grant FEEDBACK 340442. We also acknowledge the 3D-HST collaboration, who provided us with spectroscopic and photometric catalogs used to select MOSDEF targets and derive stellar population parameters. We thank Daniel Stark for insightful comments. We wish to extend special thanks to those of
Hawaiian ancestry on whose sacred mountain we are privileged to be guests.

\section{ORCID iDs}

Ryan L. Sanders (ib https://orcid.org/0000-0003-4792-9119

Naveen A. Reddy (i) https://orcid.org/0000-0001-9687-4973

Mariska Kriek (1) https://orcid.org/0000-0002-7613-9872

Brian Siana (ib https://orcid.org/0000-0002-4935-9511

Alison L. Coil @ https://orcid.org/0000-0002-2583-5894

Gene C. K. Leung (1) https://orcid.org/0000-0002-9393-6507

Irene Shivaei 다 https://orcid.org/0000-0003-4702-7561

Sedona H. Price (1) https://orcid.org/0000-0002-0108-4176

Mojegan Azadi (i) https://orcid.org/0000-0001-6004-9728

James Aird (ㄴ) https://orcid.org/0000-0003-1908-8463

\section{References}

Andrews, B. H., \& Martini, P. 2013, ApJ, 765, 140

Asplund, M., Grevesse, N., Sauval, A. J., \& Scott, P. 2009, ARA\&A, 47, 481

Belli, S., Jones, T., Ellis, R. S., \& Richard, J. 2013, ApJ, 772, 141

Calzetti, D., Armus, L., Bohlin, R. C., et al. 2000, ApJ, 533, 682

Caputi, K. I., Deshmukh, S., Ashby, M. L. N., et al. 2017, ApJ, submitted (arXiv:1705.06179)

Cardelli, J. A., Clayton, G. C., \& Mathis, J. S. 1989, ApJ, 345, 245

Chabrier, G. 2003, PASP, 115, 763

Conroy, C., Gunn, J. E., \& White, M. 2009, ApJ, 699, 486

Genzel, R., Newman, S., Jones, T., et al. 2011, ApJ, 733, 101

Gordon, K. D., Clayton, G. C., Misselt, K. A., Landolt, A. U., \& Wolff, M. J. 2003, ApJ, 594, 279

Heckman, T. M. 2002, in ASP Conf. Ser. 254, Extragalactic Gas at Low Redshift, ed. J. S. Mulchaey \& J. Stocke (San Francisco, CA: ASP), 292

Heckman, T. M., Robert, C., Leitherer, C., Garnett, D. R., \& van der Rydt, F. 1998, ApJ, 503, 646

Jones, T., Martin, C., \& Cooper, M. C. 2015, ApJ, 813, 126

Kennicutt, R. C. 1998, ARA\&A, 36, 189

Kriek, M., Shapley, A. E., Reddy, N. A., et al. 2015, ApJS, 218, 15

Kriek, M., van Dokkum, P. G., Labbé, I., et al. 2009, ApJ, 700, 221

Madau, P., \& Dickinson, M. 2014, ARA\&A, 52, 415

Maiolino, R., Nagao, T., Grazian, A., et al. 2008, A\&A, 488, 463

Mannucci, F., Cresci, G., Maiolino, R., Marconi, A., \& Gnerucci, A. 2010 MNRAS, 408, 2115

Masters, D., McCarthy, P., Siana, B., et al. 2014, ApJ, 785, 153

McLean, I. S., Steidel, C. C., Epps, H. W., et al. 2012, Proc. SPIE, 8446, 84460J

Momcheva, I. G., Brammer, G. B., van Dokkum, P. G., et al. 2016, ApJS, 225,27

Nagao, T., Maiolino, R., \& Marconi, A. 2006, A\&A, 459, 85

Onodera, M., Carollo, C. M., Lilly, S., et al. 2016, ApJ, 822, 42

Reddy, N. A., Erb, D. K., Pettini, M., Steidel, C. C., \& Shapley, A. E. 2010, ApJ, 712, 1070

Reddy, N. A., Oesch, P. A., Bouwens, R. J., et al. 2017, ApJ, submitted (arXiv:1705.09302)

Salmon, B., Papovich, C., Finkelstein, S. L., et al. 2015, ApJ, 799, 183

Sanders, R. L., Shapley, A. E., Kriek, M., et al. 2015, ApJ, 799, 138

Sanders, R. L., Shapley, A. E., Kriek, M., et al. 2016a, ApJL, 825, L23

Sanders, R. L., Shapley, A. E., Kriek, M., et al. 2016b, ApJ, 816, 23

Schmidt, M. 1959, ApJ, 129, 243

Shapley, A. E., Reddy, N. A., Kriek, M., et al. 2015, ApJ, 801, 88

Sharples, R., Bender, R., Agudo Berbel, A., et al. 2013, Msngr, 151, 21

Skelton, R. E., Whitaker, K. E., Momcheva, I. G., et al. 2014, ApJS, 214, 24

Smit, R., Bouwens, R. J., Labbé, I., et al. 2014, ApJ, 784, 58

Stanway, E. R., Eldridge, J. J., \& Becker, G. D. 2016, MNRAS, 456, 485

Stark, D. P., Schenker, M. A., Ellis, R., et al. 2013, ApJ, 763, 129

Steidel, C. C., Rudie, G. C., Strom, A. L., et al. 2014, ApJ, 795, 165

Swinbank, A. M., Bower, R. G., Smith, G. P., et al. 2007, MNRAS, 376, 479

Swinbank, A. M., Webb, T. M., Richard, J., et al. 2009, MNRAS, 400, 1121

Tacconi, L. J., Neri, R., Genzel, R., et al. 2013, ApJ, 768, 74

Tremonti, C. A., Heckman, T. M., Kauffmann, G., et al. 2004, ApJ, 613, 898

Troncoso, P., Maiolino, R., Sommariva, V., et al. 2014, A\&A, 563, A58

Turner, O. J., Cirasuolo, M., Harrison, C. M., et al. 2017, MNRAS, 471, 1280

Zahid, H. J., Kashino, D., Silverman, J. D., et al. 2014, ApJ, 792, 75 